\title{
A ROADMAP FOR STRATEGIC RESEARCH ON VIRTUAL ORGANIZATIONS
}

\author{
Luis M. Camarinha-Matos ${ }^{1}$, Hamideh Afsarmanesh ${ }^{2}$ \\ ${ }^{1}$ New University of Lisbon, PORTUGAL, cam@uninova.pt \\ ${ }^{2}$ University of Amsterdam, THE NETHERLANDS, hamideh@science.uva.nl
}

A roadmap to the necessary integrated research and technological development towards a new generation of virtual organizations is presented. In special the roadmapping methodology developed in the VOmap project is described and exemplified.

\section{INTRODUCTION}

Roadmapping became quite popular in the USA in the late 1990's, and more recently in Europe, especially in terms of technology roadmaps, as an approach to strategic planning for the future of technology in different sectors. However, a common definition for roadmapping or roadmap does not exist, and an observation of roadmaps that have been created so far indicates that there is considerable diversity among practitioners as to what constitutes a roadmap and the roadmapping techniques employed. According to some authors, roadmapping is just good planning [5]. For Robert Galvin [8]:

"A 'roadmap' is an extended look at the future of a chosen field of inquiry composed from the collective knowledge and imagination of the brightest drivers of change in that field."

As implicit above, a roadmap shall reflect the knowledge/vision of a group of experts and visionaries, but not necessarily the widest democratic consensus. In fact, the term "reaching consensus", so popular in the EC funded research circles, is even dangerous and counter productive if taken to an extreme, to a roadmap aiming for open new frontiers. Real breakthroughs in science and technology are always the result of the activity of a few visionaries, not something created by a "parliamentary decision" or "poll". Its value has to be assessed by the soundness of the arguments and its base logic, not by the number of votes it collected. In other words, the assessment of a roadmap is something that can only be done by those experts with a quite broad view and understanding of the target area.

Various types of roadmaps, with rather different scopes and level of generality, can be considered. For instance [8], [9]: 
- Science and research roadmaps

- Cross-industry roadmaps

- Industry roadmaps

- Technology roadmaps

- Product roadmaps

- Product-technology roadmaps

- Project and issue roadmaps.

The IST VOmap roadmapping project was designed to identify and characterize the key research challenges needed to fulfill the vision, required constituency, and the implementation model for a comprehensive European initiative on dynamic virtual organizations (VO). VOmap is clearly positioned at the strategic science and research roadmapping level. Therefore, the expected result is not a roadmap focused on a particular product or technology (the most usual type of roadmaps), but rather the definition of a strategic research program for virtual organizations.

A good number of roadmaps are now publicly available (see [1], [2], [3], [4], [5], [6], [7], [11] for instance). However, in spite of the growing interest in roadmapping, there is not yet a real systematic roadmapping or even visioning methodology defined.

Even for one of the main (and well known) steps, the "gap analysis" there is no clear procedure or set of tools available. The known approaches, such as the "SWOT analysis", do not directly apply here and cannot help, due to the fact that:

1. they are not "procedural" methodologies that can be simply applied to this case, and

2. the target context of these approaches are different than what is needed to be generate here for roadmapping, which is an ordered set of transition steps and a time line.

Furthermore, roadmap studies performed so far indicate a long-term procedure (longer than one year), led by a group of experts (roadmap leading team or coordinators), and involving a very large group of experts from industry, research, and academia more than 100).

Therefore, although we sought inspiration from a large number of roadmap examples, VOmap had to develop its own innovative working methodology and procedures for roadmapping, which is summarized and exemplified in the next sections. Furthermore VOmap builds on preliminary results from two other IST networks, the THINKcreative and VOSTER projects. THINKcreative aims at identifying and characterizing emerging organizational collaborative forms and their required infrastructures, modeling and application tools, as well as the corresponding socio-organizational needs for the next 5, 10 and 20 years [12]. VOSTER represents an effort to cluster major European VO projects, systematize the state of the art, and contribute to the harmonization of models and infrastructures [13].

\section{BASELINE AND VISION}

Two important elements for a strategic research roadmap are the state of the art (baseline) and the vision of desired VO frontier. Figure 1 below represents the goal 
of VOmap in a nutshell, which is to develop a Roadmap, identifying all necessary transition steps, involving research, development and implementation, to reach the future desired state of the Virtual Organizations.

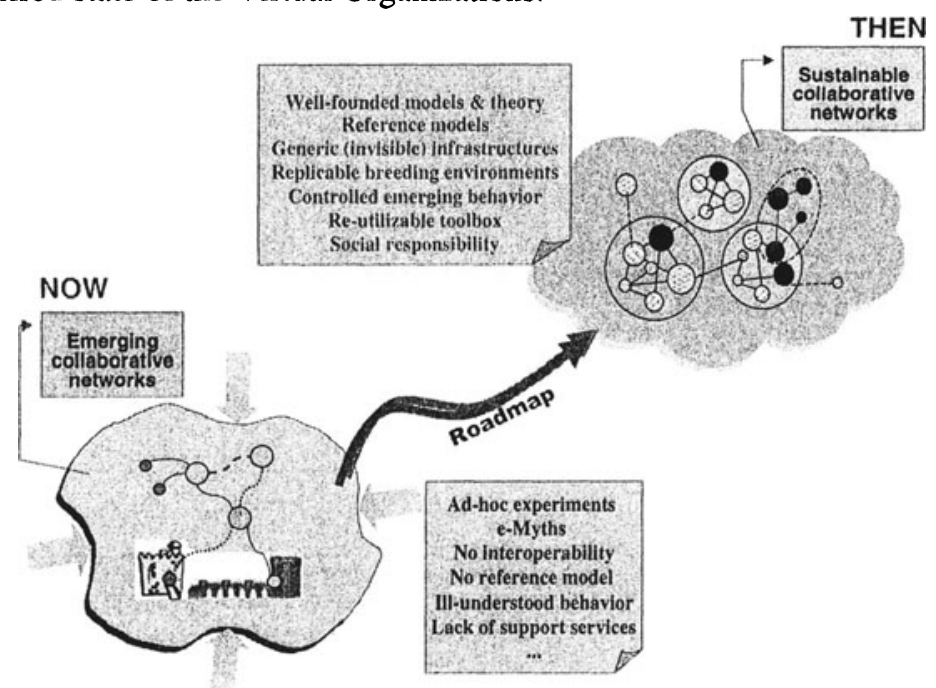

Figure 1. VOmap in a nutshell

The baseline for virtual organizations in Europe can be summarized in the following statements:

- Research on VO has created a critical mass and European-wide intuitive understanding of the area.

- Basic supporting infrastructures and relevant technologies are well represented, but the developments are often focused on particular needs and based on ad-hoc experiments, hardly re-utilizable.

- Generic functions or harmonization of achievements are addressed only in a few projects.

- Efforts on general plug-and-play architecture and interoperability are also to a large extent missing.

\section{iConsequently, no generally accepted reference model or} interoperability base are available.

- Although several disciplines are concerned, the main focus has been on the ICT infrastructure. Research on social/organizational, including management, is mainly focused on best practice. Integration with technological development and impacts on structures are not covered. In addition little research is focused on the social and organizational issues created by VOs

Furthermore, the rapid evolution of the Internet-related technologies and the fast proliferation of tools have facilitated the dissemination of myths and creation of expectations that are hardly confirmed in practice. There is, therefore, an urgent need to establish the foundations for a new research and development phase based on a clear strategic vision.

Vision building is not a mechanism to foresee the future. Clearly, the entire future of collaborative networks and VOs in Europe can never be fully predicted. However significant market trends, technology trends, the state of the art, and consolidated baselines are either already in evidence or can be investigated, which 
can be used as the resources, mechanisms, and/or constraint, towards establishing $a$ vision for desired collaborative networks of the future.

Furthermore, although not a task in VOmap, the elaboration and characterization of plausible future scenarios for collaborative network organizations and professional virtual communities were carefully taken into account. The considered scenarios were either originated in different forums (e.g. THINKcreative, COVE, CE-NET) [12] and a few in VOmap, or appeared in well-referenced and large international initiatives, backed up by a huge number of experts' validation. The VOmap vision is stated as:

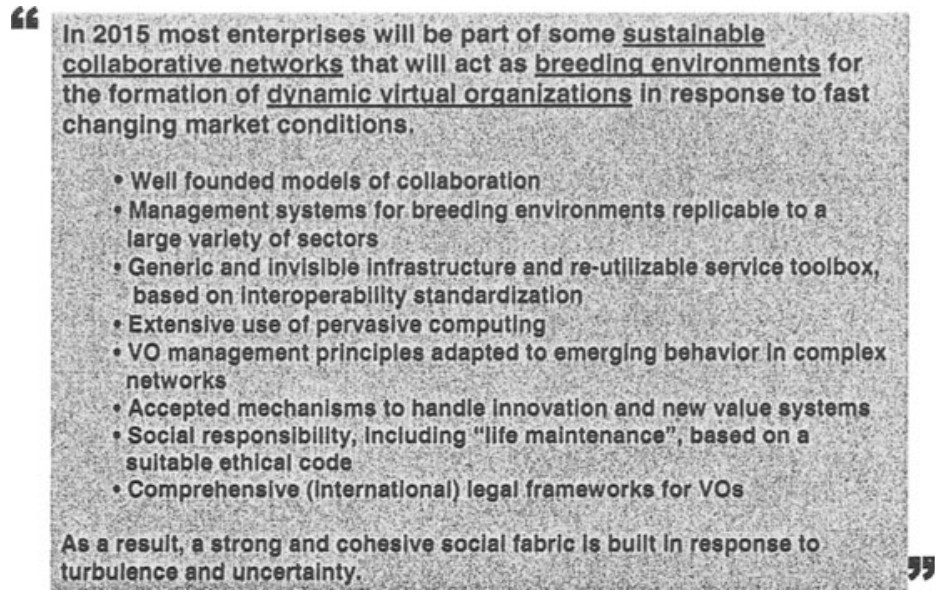

The above vision statement creates new opportunities for businesses, large and small, to become both more competitive in future global market and more innovative and risk taking. On the other hand it implicitly suggests the main areas needing attention from other social bodies and institutions in order to reach this desired state. Namely: 1) those areas in which the governments and regulatory bodies must invest more attention, and 2) those areas that require more research and development.

An important contribution of VOmap, which results from understanding the VO phenomena as a multi-disciplinary one, was the instantiation of the vision according to five focus areas.

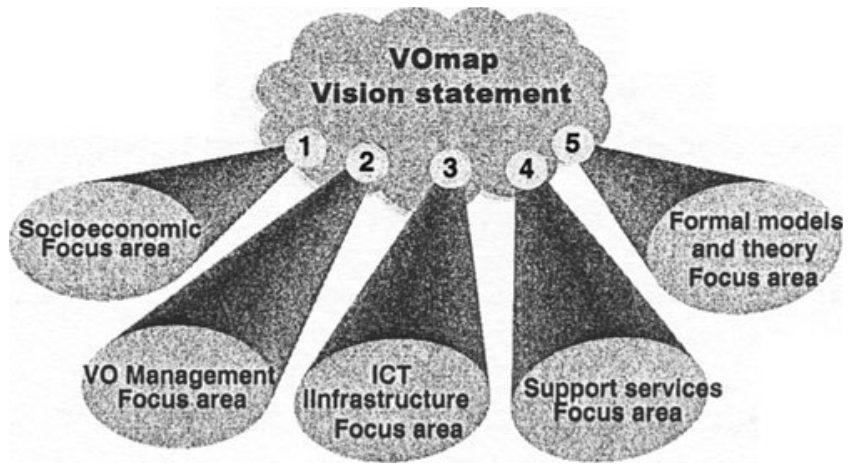

Figure 2 - VOmap focus areas 
In addition to the vision instantiation, for each focus area the key facets or mechanisms that characterize the vision are identified as shown in next figure.

Vision according to the socio-economic focus area:

\begin{tabular}{|c|c|}
\hline $\mathrm{VE}_{1}$ & $\begin{array}{l}\text { People being prepared and supported to work as employees or professionals in } \\
\text { enterprise networks or other virtual organization settings }\end{array}$ \\
\hline $\mathrm{VE}_{2}$ & $\begin{array}{l}\text { New mechanisms and professional communities to provide for human sense of } \\
\text { belonging, long-lasting relationships and stability (social responsibility) }\end{array}$ \\
\hline $\mathrm{VE}_{3}$ & $\begin{array}{l}\text { New institutions and models to support "life maintenance", e.g. social security and } \\
\text { personal training and development }\end{array}$ \\
\hline $\mathrm{VE}_{4}$ & $\begin{array}{l}\text { Support for companies by enabling institutions and services to set-up, enter and } \\
\text { develop virtual organizations regionally and internationally }\end{array}$ \\
\hline $\mathrm{VE}_{5}$ & $\begin{array}{l}\text { Comprehensive and transparent legal framework, specially in the case of institutional } \\
\text { collaboration }\end{array}$ \\
\hline $\mathrm{VE}_{6}$ & Regional assets and cultural identity leveraged and preserved \\
\hline $\mathrm{VE}_{7}$ & $\begin{array}{l}\text { Well founded understanding of social and socio-economic processes and } \\
\text { developments in the context of networked economies }\end{array}$ \\
\hline
\end{tabular}

Vision according to the VO management focus area:

\begin{tabular}{|c|c|}
\hline \multicolumn{2}{|r|}{$\begin{array}{l}\text { Well-defined business models will be developed to allow the systematic VO management, } \\
\text { namely to act in regards to planning, control, organization and leadership, taking into account } \\
\text { the importance of social mechanisms in multi-Interest collaboration networks, as well as the } \\
\text { transitional nature of VO. }\end{array}$} \\
\hline $\mathrm{VM}_{1}$ & Wide understanding of brokerage and pro-active approach to VO formation \\
\hline $\mathrm{VM}_{2}$ & $\begin{array}{l}\text { Understanding of strategy development and value creation in VO and the subsequent } \\
\text { establishment of VO planning and performance assessment mechanisms }\end{array}$ \\
\hline $\mathrm{VM}_{3}$ & $\begin{array}{l}\text { Clear mechanisms for leadership and human resource practices, conflict management } \\
\text { and participation with regard to shared and distributed decision-making in "multiple- } \\
\text { objective" collaboration spaces }\end{array}$ \\
\hline $\mathrm{VM}_{4}$ & $\begin{array}{l}\text { Defined organization principles for seamless flow of responsibility, ownership, } \\
\text { knowledge and benefits along the VO life cycle and across VO boundaries, in relation } \\
\text { to customers, other networks and third parties }\end{array}$ \\
\hline $\mathrm{VM}_{5}$ & $\begin{array}{l}\text { Mechanisms and institutions to foster long / short term collaboration with the aid of } \\
\text { "soft" trust-building support on internal and external level (e.g. code of ethics) as well } \\
\text { as complementary "hard" incentive schemes }\end{array}$ \\
\hline
\end{tabular}

Vision according to the ICT infrastructure focus area:

The ICT infrastructure will be developed as a transparent, low-cost, and easy to use enabler of
collaborative behaviors in networked organizations.
Mechanisms:
$\mathrm{VI}_{1} \quad$ Technology-independent reference architecture for the horizontal infrastructure
$\mathrm{VI}_{2} \quad$ Provide support for federated information and resources management
$\mathrm{VI}_{3} \quad$ Flexible control mechanisms supporting the implementation of a large variety of

$\mathrm{VI}_{4} \quad$ behaviors
$\mathrm{VI}_{5} \quad$ Full e-transaction security and privacy is guaranteed
$\mathrm{VI}_{6} \quad$ "Configure yourself" philosophy (user "programmable" infrastructure)


Vision according to the support services focus area:

\begin{tabular}{|c|c|}
\hline \multicolumn{2}{|r|}{$\begin{array}{l}\text { IT support services will be developed to assist VO brokers, management and employees with } \\
\text { their tasks for setting-up, operating and dissolving virtual organizations. } \\
\text { The tools are embedded in flexible architectures suited for different types of virtual } \\
\text { organizations, driven by business, social, legal, etc. needs and are easy to use and provide a } \\
\text { well balanced approach between human support and business process automation. } \\
\text { Mechanisms: }\end{array}$} \\
\hline $\mathrm{VS}_{1}$ & $\begin{array}{l}\text { Management of breeding environment (e.g. definition, behaviour, membership, rules, } \\
\text { rights, responsibilities, business interoperability) }\end{array}$ \\
\hline $\mathrm{VS}_{2}$ & $\begin{array}{l}\text { VO creation framework (choices of automatic / semi-automatic or search assisted by } \\
\text { the breeding environment's manager) }\end{array}$ \\
\hline $\mathrm{VS}_{3}$ & Coordination/management of highly distributed activities (human assisted) \\
\hline $\mathrm{VS}_{4}$ & $\begin{array}{l}\text { nagement, assessment tools, performance measuring and mecha } \\
\text { and experience collection }\end{array}$ \\
\hline & isms for traceability and for handling post-cooperation IPRs an \\
\hline
\end{tabular}

Vision according to the formal models and theories focus area:

Decision-making in all phases of the VO life cycle is based on well argued and verified models and methodologies, which are the basis for the ICT-based support for business and organizational development and operation, as well as the base for education, training, and active operation of VOs.

Mechanisms:

$V_{1} \quad$ Established formal foundation to guarantee VOs effectiveness (performance management), better decision-making, incremental learning from past experience, and minimized operating problems via clear commitments

$V_{2}$ The $V O$ research area is recognized (and respected) as a scientific discipline

$\mathrm{VF}_{3} \quad$ Generic modeling of the VO (structure and behavior) as a top-down approach addresses e.g. VO configuration, roles and responsibilities, coordination, distributed process management, general agreements and contract

$\mathrm{VF}_{4} \quad$ Generic modeling of VO members' behavior as a bottom-up approach addresses e.g. contributed assets, accepted responsibilities, acquired rights, individual commitments and contract

$V F_{5} \quad$ Discipline-specific formal models are defined

$V_{6} \quad$ Models interoperability (generic and discipline-specific) are defined

Figure 3 - Vision instantiations

For space limitations, not all focus areas can be fully described in this paper. Therefore, one of them, the "formal models and theory focus area", will be used to illustrate the various phases of the VOmap approach.

\section{STEPS TOWARDS A ROADMAP}

Considering the current state of collaborative networks and virtual organizations in Europe, in order to elaborate a list of actions that will be likely to help achieving the desired vision, the following steps or tasks are proposed in the VOmap roadmapping methodology: 
Task 1. Filling the GAP: Where we are - to - Where we wish to go

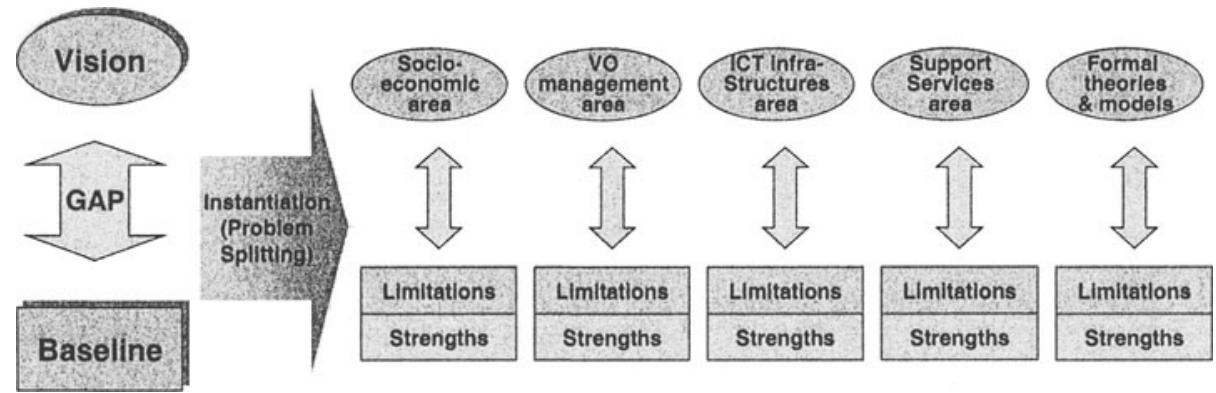

Figure 4 - Vision and baseline instantiations for gap analysis

According to the VOmap approach, for the gap analysis, the detailed characterization and essence of the baseline ("where we are") in comparison to the vision (where we wish to go) is achieved by the two main facets of the current states: Strengths and Limitations. And again, similarly to the vision, this baseline characterization and gap analysis shall also be divided and approached according to the specificities of each of the main focus areas. Example:

\section{Baseline:}

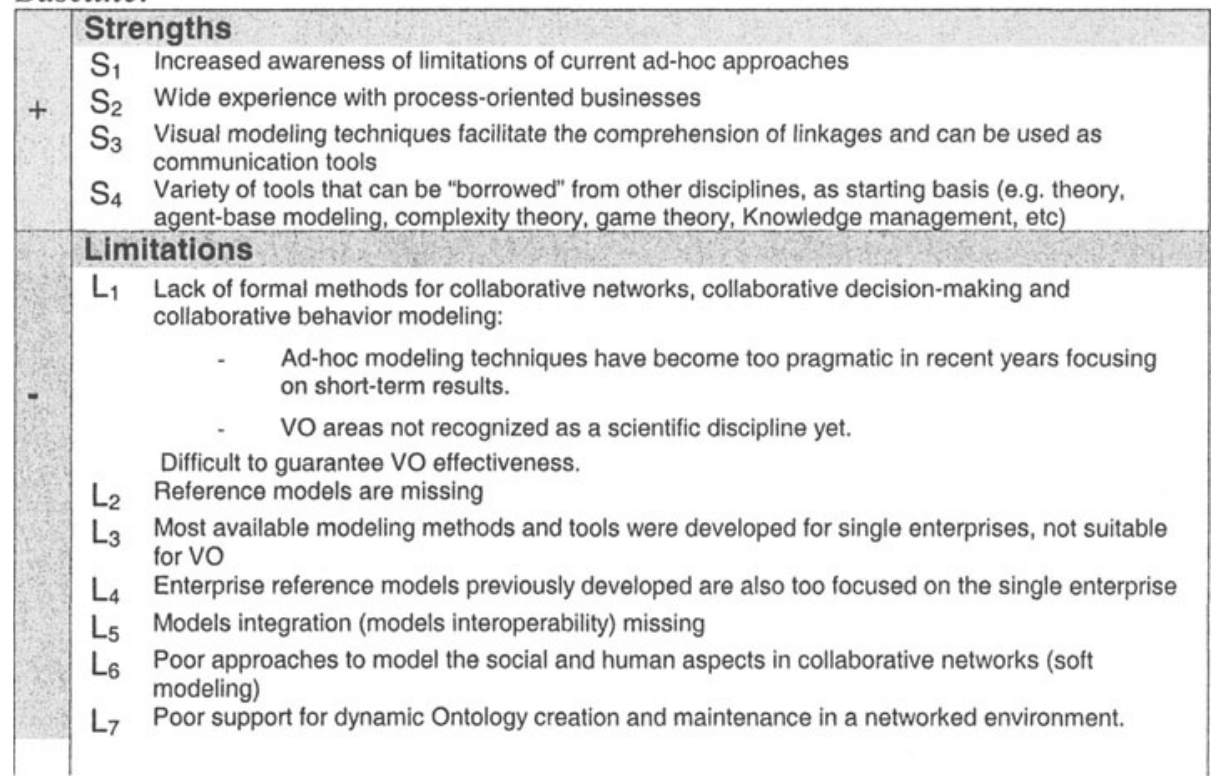

Figure 4 - Example of strengths and limitations

After reaching the gap analysis instantiations, we can generate the "Influence maps" (matrix or graph) to represent the positive / negative influences of the baseline to the achievement of the vision. 
A "strength" is likely to have a positive effect (moderate or high) on some of the vision facets. A "Limitation" on the other hand will have a negative influence (moderate or high) on the achievement of some of the vision facets.

These influence maps provide some qualitative analysis of the state of potentials and barriers on the road to reach the vision. Therefore, these maps will help experts to observe how difficult or easy it is to achieve a vision facet.

Example:

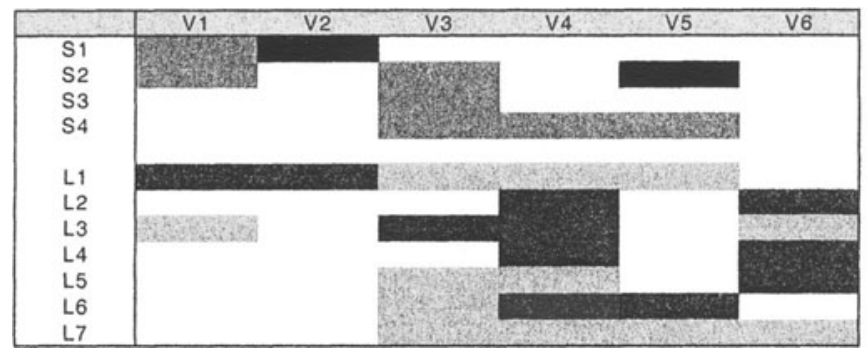

Positive influence

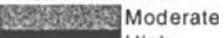
High

Negative influence

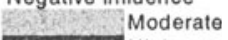

Figure 5 - Example of influence map - matrix form

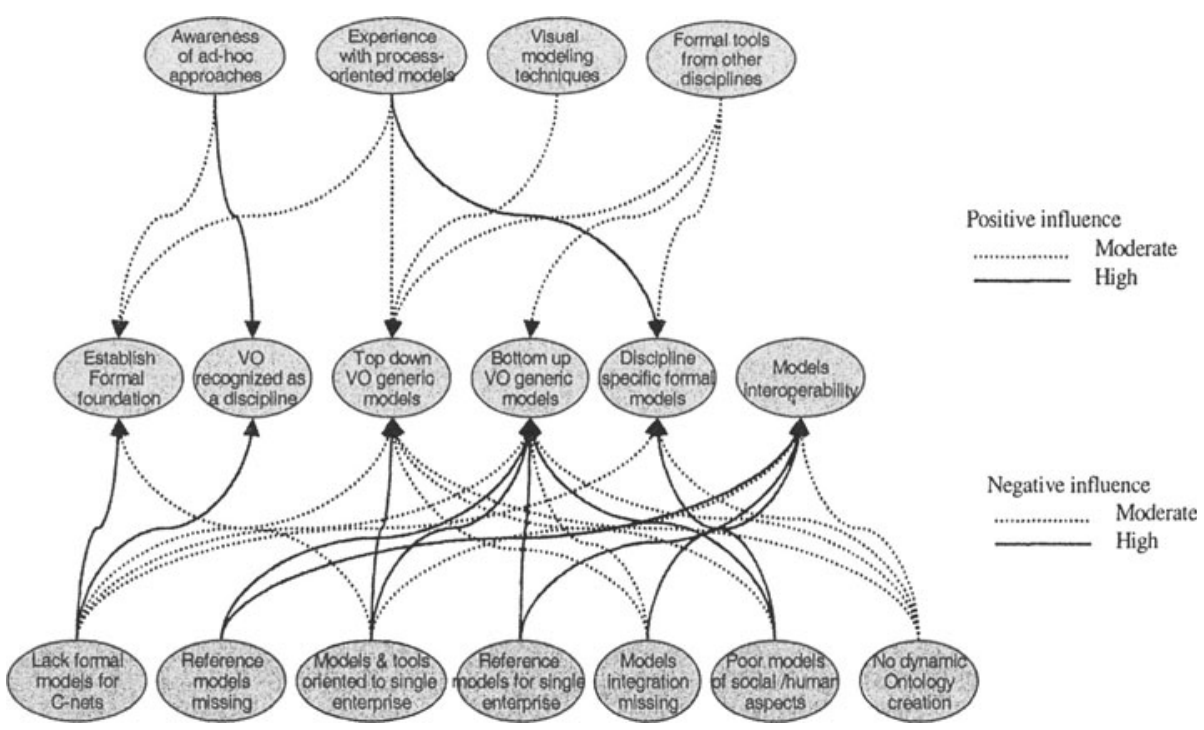

Figure 6 - Example of influence map - graph form

\section{Task 2. Propose a plan of actions (a set of unordered transition steps)}

Due to its complexity here we do not need to necessarily suggest one procedure on how this step can be achieved. Several different ways could be pursued. For instance, using all the results achieved in previous steps, an expert in the field may pursue his/her systematic (difficult to devise) or "ad-hoc" approach to configure and propose these actions, or even a group of experts may brainstorm, which is in fact what can for instance be achieved in a workshop of experts. Therefore, no matter 
how it is done, the following types of results must be generated. Namely, a list of actions (un-ordered transition steps) must be designed for each main focus area.

\section{Example:}

\begin{tabular}{|c|c|}
\hline \multicolumn{2}{|c|}{ Vision: } \\
\hline$V_{1}$ & $\begin{array}{l}\text { Established formal foundation to guarantee VOs effectiveness (performance } \\
\text { management), better decision-making, incremental learning from past experience, } \\
\text { and minimized operating problems via clear commitments }\end{array}$ \\
\hline$V_{2}$ & The VO research area is recognized (and respected) as a scientific discipline \\
\hline$V_{3}$ & $\begin{array}{l}\text { Generic modeling of the VO (structure and behavior) as a top-down approach } \\
\text { addresses e.g. VO configuration, roles and responsibilities, coordination, } \\
\text { distributed process management, general agreements and contract }\end{array}$ \\
\hline $\mathrm{V}_{4}$ & $\begin{array}{l}\text { Generic modeling of VO members' behavior as a bottom-up approach addresses } \\
\text { e.g. contributed assets, accepted responsibilities, acquired rights, individual } \\
\text { commitments and contract }\end{array}$ \\
\hline$V_{5}$ & Discipline-specific formal models are defined \\
\hline$V_{6}$ & Models interoperability (generic and discipline-specific) are defined \\
\hline
\end{tabular}

Considering Strengths \& Limitations of the Baseline

$A_{1} \quad \begin{aligned} & \text { Establish a formal theoretical foundation for modeling dynamic } \\ & \text { collaborative networks } \\ & A_{2}\end{aligned}$
$\begin{aligned} & \text { Define basic formal reference models (including ontologies) for } \\ & \text { collaborative networks at general and focused-area levels }\end{aligned}$
$A_{3} \quad \begin{aligned} & \text { Elaborate soft modeling approaches and soft models to both handle } \\ & \text { incomplete / imprecise knowledge and capture the social/human aspects } \\ & \text { in collaborative networks }\end{aligned}$
$A_{4} \begin{aligned} & \text { Devise mechanisms for evolution and maintenance of reference models } \\ & \text { for collaborative networks }\end{aligned}$
$A_{5} \begin{aligned} & \text { Elaborate approaches for models interoperability, supporting multiple } \\ & \text { modeling perspectives (e.g. structure, behavior) at generic and focused } \\ & \text { area levels }\end{aligned}$

Figure 7 - First proposition of research actions for formal models and theories

\section{Task 3. Verify the planned actions}

Since the approach taken in task 2 is most likely an ad-hoc approach, in order to validate the proposed actions, here in this task we consider to apply two main verifications means:

Criterion 1- Check how the proposed actions "contribute" to fulfill the vision.

Criterion 2- Check how "feasible" these actions are, considering the two facets

of the baseline, i.e. the positive and negative factors.

In both cases we also like to come up with some "qualitative values".

In each focus area, the results of Criterion 1 constitute many to many relationships between the Actions and the Vision statements. Namely, it would indicate for each action, the exact vision statement that it influences, and vice versa about the coverage. We will also rate the qualitative level of relevance or coverage for each Action (Moderate, High, Very High).

The results of criterion 2 would be the feasibility check of the Actions against the 2 facets (positive and negative) of the baseline, and this time rated by the qualitative level of their difficulty (i.e. Moderate, Hard, Very Hard). 
Example:

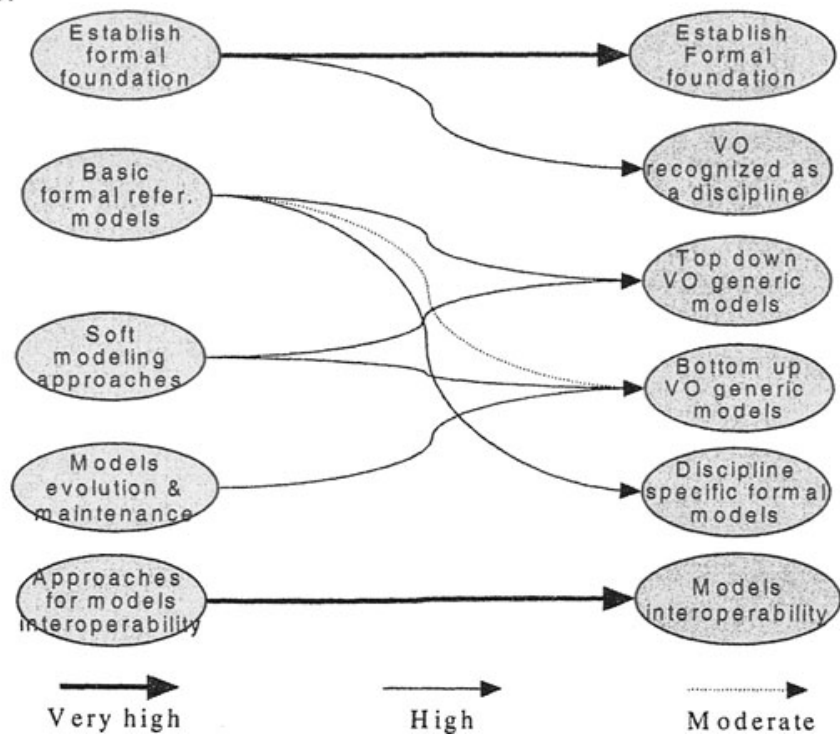

Figure 8-Example of contributions and feasibility of proposed actions - graph form

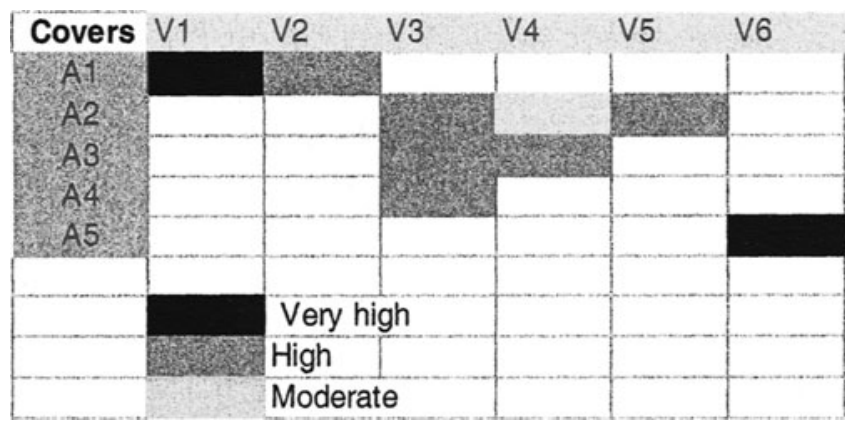

Figure 9 - Example of contributions and feasibility of proposed actions - matrix form

\begin{tabular}{|c|c|c|c|c|c|c|c|c|c|c|c|c|}
\hline & S1 & S2 & S3 & S4 & L1 & $\angle 2$ & L3 & L4. & $\mathrm{L} 5$ & L6 & L7 & Difficulty \\
\hline At: & & & & & 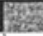 & & & & & & & Moderate \\
\hline A2 & & & & & & & & & & & & Mod/hard \\
\hline$A 3$ & & & & & & & & & & & & Hard \\
\hline A4 & & & & & & & & & & & & Hard \\
\hline$A 5$ & & & & & & & & & & & & Mod/Hard \\
\hline & & & & & & & & & & & & \\
\hline & & \multicolumn{3}{|c|}{ Strong help from } & & & \multicolumn{3}{|c|}{ Strongly limited by } & & & \\
\hline & & \multicolumn{3}{|c|}{ Moderate help from } & & & \multicolumn{3}{|c|}{ Moderately limited by } & & & \\
\hline & & \multicolumn{3}{|c|}{ Limited help from } & & & \multicolumn{3}{|c|}{ Partially limited by } & & & \\
\hline
\end{tabular}

Figure 10 - Actions feasibility check for the formal theories and models focus area 


\section{Task 4. Time planning and other characterization of actions}

Here we try to identify the importance, to add the time plan, and to better characterize the generated actions in Task 3 , by adding the main relevant features such as resources, required skills, etc.

\section{Task 5. Finalize the definition of the roadmap chart}

Here, taking the results of Task 4 above on the actions, we check the interdependencies among them and produce the time-based transition steps for each focus area, as well as among different focus areas. As such:

FIRST: This task focuses on the careful identification of the interdependencies and links among different actions within each focus area, by also paying careful attention to the time schedule of these actions. Here a partial roadmap chart will be achieved for each focus area, by the generation of a chart, representing an ordered set of transition steps and the time line for these steps.

SECOND: These partial roadmap charts will be inter-related, by careful consideration of how the transition steps in one focus area will relate to those of the other focus area.

In this inter-linking / harmonization exercise among all 5 focus areas, sometimes the logical and/or the time order among different transition steps, from different focus areas, necessitates a change in the partial roadmap chart for the focus areas, generated before. For example, in the following Figure, the transition step A3 (from Focus Area A) needs the results of the transition step B2 (from Focus Area B), therefore either A3 must move forward in time (e.g. locate below A4) or B2 must move backward in time (e.g. above B1) in order to guarantee this inter-linking.

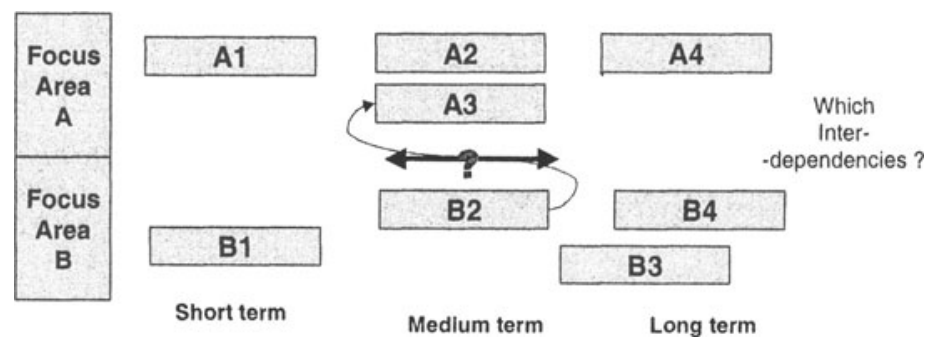

Figure 11 - Roadmap presentation template

At the end of this procedure, a first roadmap can be summarized in a diagram as shown in Fig. 12. 


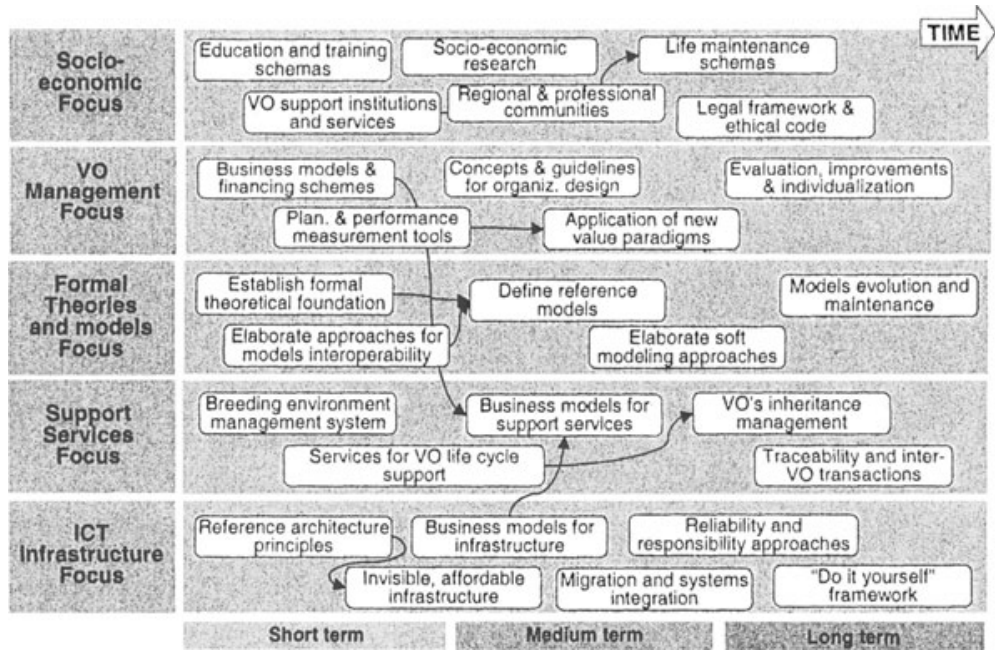

Figure 12 - The first draft roadmap

After the elaboration of a first draft roadmap it is important to validate and refine it with the participation of a wider group of experts. For this purpose, VOmap has established a Support Group of more than 40 organizations from the various European regions. A key mechanism to get a strong involvement of this Support Group was the organizations of a number of regional meetings / discussion panels, which took place in Portugal, Netherlands, Germany (2), Switzerland, and Finland (2). It shall be noted that SMEs, although the potential major beneficiaries of the VOmap strategic goals, have difficulties in getting involved as full partners in a roadmap design process, mainly due to the involved "bureaucracy" of this type of initiatives. Nevertheless many SMEs are quite willing to get committed to participate in discussions and interaction with other experts. The regional / local meetings proved to be a very effective mechanism to get the participation of SMEs that usually are not available to attend large meetings abroad. With this distributed consultation mechanism a very large number of enterprises and other organizations, that otherwise would be out of this planning process, got actively involved in the roadmap design. Complementarily a number of other well-known experts from Australia, Japan, Brazil, Mexico and Canada were also invited to review the roadmap via email and suggest improvements.

Unlike other roadmapping approaches that try to perform all steps of the process through workshops involving a large number of participants, the VOmap approach was to first resort to its core members (small number) to elaborate a draft roadmap and only then submit it to a refinement process via the regional workshops. This approach proved to be very effective under the time and resources constraints of the project. All workshops could be very up to the point and the feedback received from participants was very positive.

As a last step, the integration and refinement of the roadmap was performed taking into account the results of the regional workshops. When defining the final version of the roadmap three phases are considered: 1) R\&D, 2) Trials, and 3) Broad deployment. Fig. 14 shows the integrated roadmap. It shall be noted that the three mentioned phases do not happen in a strict sequential order, but they rather (partially) overlap (Fig. 13). 


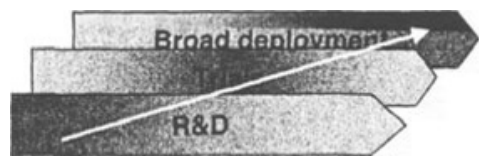

Figure 3 - Implementation phases and their "intensity" periods

Therefore, an R\&D block in Fig. 14 shall be interpreted as this being a phase were the main emphasis is on R\&D although some trials and even broad deployment of partial results might start in parallel. The interdependencies among actions are not shown.

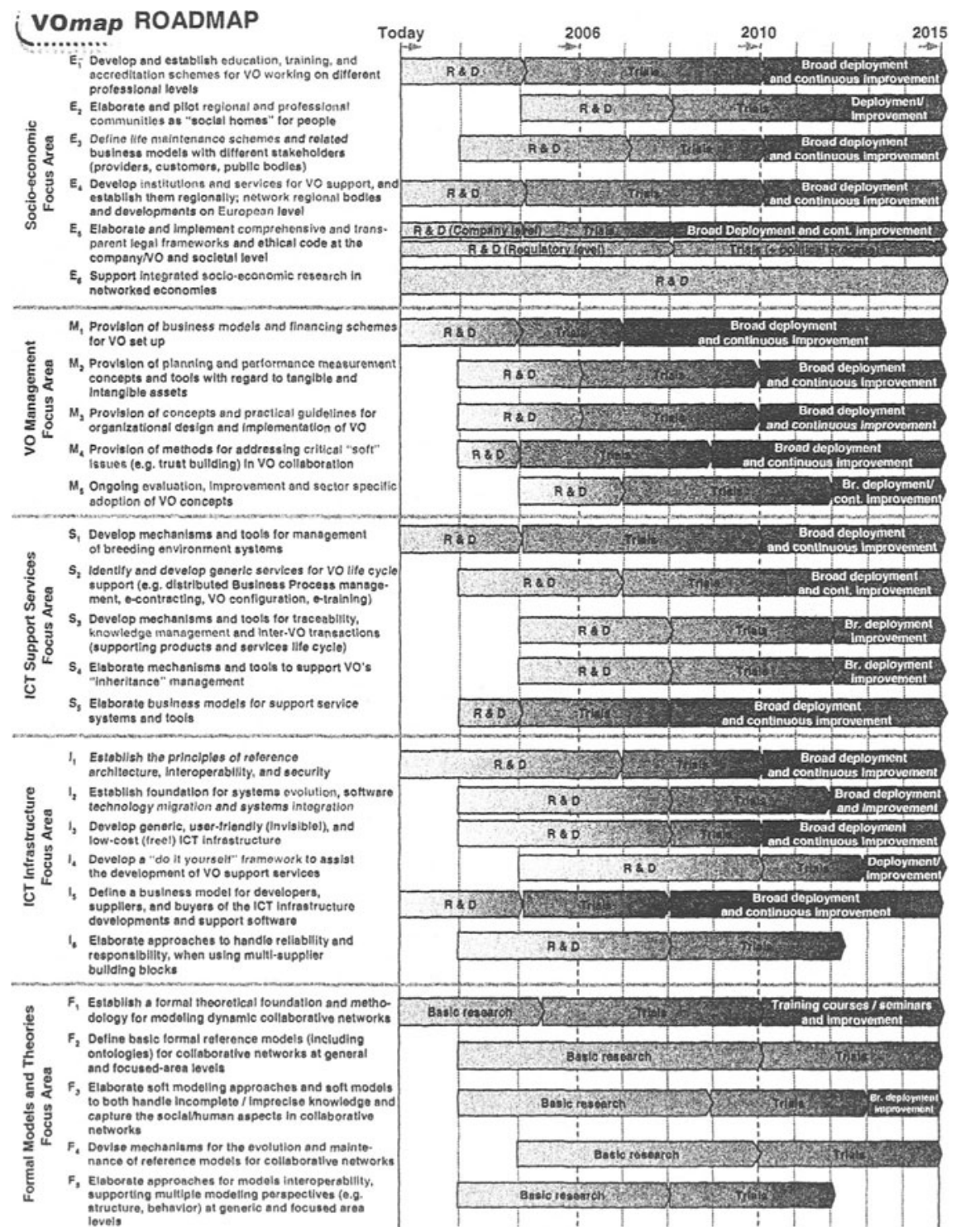

Figure 13 - The VOmap roadmap for advanced virtual organizations 


\section{CONCLUSIONS}

Collaborative networks in general, and virtual organizations in particular, provide a basis for competitiveness, world-excellence, and agility in turbulent market conditions. Reinforcing the effectiveness of collaborative networks and creating the necessary conditions for making them an endogenous reality in the European industrial landscape, mostly based on SMEs, is a key challenge. During the last decade, and in parallel with the development and spreading of Internet technologies, traditional collaboration networks have found new leveraging tools and the new collaborative business forms have emerged. In terms of research, there has been significant activity during the last decade especially in Europe in the area of Virtual Organisations. However, these initiatives correspond to fragmented research and in most cases, due to the funding criteria, target very short-term objectives, focused on solving a specific problem, and are too biased by "fashionable" short-life technologies. It is therefore necessary to start a new phase of research and development on virtual organizations.

As a response to this situation, VOmap developed an effective roadmapping methodology and proposed a strategic research roadmap focused on creating the necessary strong foundations and mechanisms for establishing the most advanced collaborative and network-based industry society in Europe.

Acknowledgements. The work described in this paper was funded in part by the European Commission through the IST VOmap project. Partial inputs were also received from the IST THINKcreative and VOSTER projects. The authors thank the collaboration of their partners in the mentioned projects.

\section{REFERENCES}

1. Vision 2020: The lighting technology roadmap, US Department of Energy, http://www.eere.energy.gov/buildings/vision2020/pdfs/lighting roadmap.pdf

2. The Global Manufacturing Enterprise in 2020: A Perspective, Jonathan Gerhard, Georgia Institute of Technology, http://www.srl.gatech.edu/people/jonathan/ASME2020Gerhard.pdf

3. Fundamentals of technology roadmapping, Sandia National Laboratories, http://www.sandia.gov/Roadmap/home.htm\#what01

4. [Product] Roadmapping, Gerrit Muller, http://www.extra.research.philips.com/natlab/sysarch/RoadmappingSlides.pdf

5. A roadmapping perspective: Science-driven technologies, R. E. Albright, http://www.albrightstrategy.com/papers/A Roadmapping Perspective-Albright-09-26-02.pdf

6. Understanding roadmapping, Industry Canada, http://strategis.ic.gc.ca/epic/internet/intrmcrt.nsf/vwGeneratedInterE/rm00057e.html

7. Research agenda for the semantic grid: A future e-science infrastructure, D. Roure, N. Jennings, N. Shadbolt, http://www.semanticgrid.org/html/semgrid.html

8. Methods in science roadmapping: How to plan research priorities, David R. MacKenzie, Sam Donald, Mike Harrington, Robert Heil, T. J. Helms and Daryl Lund, University of Maryland, www.escop.msstate.edu/archive/roadmap-methods.doc

9. An overview of roadmapping, J. Browne, CIMRU (slides), 2002.

10. Technology roadmapping: linking technology resources to business objectives, R. Phaal, C. Farrukh, D. Probert, University of Cambrigde, Nov. 2001.

11. The future of KM: Research recommendations, European KM Forum, Working paper, 2002.

12. THINKcreative Interim Green Report on New Collaborative forms and their needs, L.M. CamarinhaMatos, H. Afsarmanesh (Ed.s), THINKcreative report Sep 2002.

13. L. M. Camarinha-Matos, K. Menzel, T. Cardoso, "ICT support infrastructures and interoperability for VOs", Deliverable D44, VOSTER. March 2003. 